\title{
Transverse magneto-optical Kerr effect of Fe at the Fe $3 p$ threshold
}

\author{
M. Pretorius, J. Friedrich, A. Ranck, M. Schroeder, J. Voss, and V. Wedemeier \\ Universität Hamburg, II. Institut für Experimentalphysik, Luruper Chaussee 149, D-22761 Hamburg, Germany \\ D. Spanke, D. Knabben, I. Rozhko, H. Ohldag, F. U. Hillebrecht, and E. Kisker \\ Institut für Angewandte Physik, Heinrich-Heine-Universität Düsseldorf, D-40225 Düsseldorf, Germany
}

(Received 31 December 1996)

\begin{abstract}
We have studied the transverse magneto-optical Kerr effect of a $\mathrm{Fe}[(5.7 \% \mathrm{Si}) 001]$ sample and of ultrathin films of Fe evaporated onto $\mathrm{Ag}(001)$ in the soft-x-ray regime at the $3 p$ core level threshold of Fe. We test the relationship between the asymmetries in the reflectivity and in the total photoyield which have been measured simultaneously. [S0163-1829(97)01822-5]
\end{abstract}

Magneto-optical effects are used in the visible part of the spectrum for magnetic domain imaging. The most important ones are the polar and the longitudinal Kerr effects which affect the polarization of the reflected light beam. Less frequently used is the transverse magneto-optic Kerr effect which refers to the dependence of the reflected intensity on the sense of the magnetization in the transverse scattering geometry, i.e., when the magnetization is directed perpendicular to the scattering plane. In a continuum view of the magnetic medium, these effects are related to the finite offdiagonal elements of the dielectric tensor. ${ }^{1}$ To first order these depend linearly on the magnetization $(M)$. In the visible part of the spectrum the transverse magneto-optical effect is very small and hence the polar and the longitudinal Kerr effects which give a strong magnetic contrast using the crossed-polarizer technique are used. It was shown recently that at core level thresholds the magneto-optical effects are very strong. This was shown first for the Fe $2 p$ level $^{2-4}$ and subsequently for the Co $3 p$ level. ${ }^{5}$ Highly efficient and tunable analyzers are not yet available for the soft-x-ray regime to accomplish domain imaging using the longitudinal or polar Kerr effect. However, it appears to be feasible to extend the concept of element-specific imaging using a scanningtype microscope with tunable primary photon energies ${ }^{6,7}$ towards element-specific imaging of magnetic domains by exploiting the huge transverse magneto-optical Kerr effect ( $\mathrm{T}$ MOKE) at the core level thresholds. The relation between the compositional distribution and the magnetization of more complex materials which are used, e.g., in magneto-optical storage and modern permanent magnets can thereby be investigated. The surface sensitivity can be altered in principle by varying the angle of incidence. The $3 p$ core levels of the transition metals are at binding energies of about $50 \mathrm{eV}$ and the reflectivity at about $15^{\circ}$ grazing incidence angle is large enough to accomplish also magnetic domain imaging when an intense soft-X-ray beam is available as, e.g., from synchrotron radiation facilities equipped with undulator beam lines. At the $2 p$ binding energies sizable reflectivity is only obtained at incidence angles very close to grazing, which makes imaging in reflection very difficult. The existence of the transverse MOKE implies a sizable dichroism in the total photoyield. This kind of magnetic dichroism has been used for imaging magnetic domains by means of photoelectron microscopy, employing a secondary electron emission microscope (PEEM). ${ }^{5}$

In the present publication, we report on new data on the transverse MOKE and the dichroism in the total photoyield obtained for the Fe $3 p$ core level. Whereas previous data ${ }^{3,5}$ had been obtained on ultrathin films, we present here data obtained from a bulk Fe(5.7\% Si)(001) sample. Furthermore, we show data obtained from ultrathin Fe films grown on $\operatorname{Ag}(001)$ as functions of film thickness and incidence angle. Finally, we investigate the relation between the transverse MOKE and the magnetic dichroism in the total photoyield.

The experiment on the $\mathrm{Fe}(5.7 \% \mathrm{Si})$ sample was performed at the VUV reflectometer station on the bending magnet beam line G1 at HASYLAB. The sample was a highly polished picture-frame-shaped single crystal. The magnetization could be reversed by a coil wound around one of its legs. The $p$-polarized synchrotron radiation was incident onto the sample at angles of $65^{\circ}$ and $69^{\circ}$. The reflected intensity was measured by means of a photodiode. A second experiment, on ultrathin Fe films on $\operatorname{Ag}(001)$, was carried out on the crossed undulator beam line U2 at the BESSY storage ring in Berlin. The Fe had been evaporated in situ onto an $\mathrm{Ag}(001)$ sample in $1 \times 10^{-8}$ mbar vacuum.

On the bulk sample, a large T-MOKE effect is observed without prior cleaning, i.e., without removing the oxide layer. Figures 1(a) and 1(b) show the reflectivity at $65^{\circ}$ incidence angle in the vicinity of the Fe $3 p$ threshold and the transverse MOKE asymmetry, respectively. The asymmetry is defined as

$$
I_{\mathrm{asy}}=\frac{I_{1}-I_{2}}{I_{1}+I_{2}},
$$

where $I_{1}$ and $I_{2}$ denote the reflectivities for two magnetization directions. The reflectivity exhibits a strong dip in the vicinity of the core level in the region $\pm 10 \mathrm{eV}$ around the excitation threshold. The asymmetry is very small out of the resonance region and shows a dip at $53 \mathrm{eV}$ and a peak at 55 eV. The p.-p. asymmetry amounts to $\sim 10 \%$. It depends strongly on the incidence angle and increases to $17 \%$ at $60^{\circ}$. Despite the fact that the sample surface is covered by an oxide layer the T-MOKE signal is quite large. The relatively weak influence of the surface oxide layer can be explained 


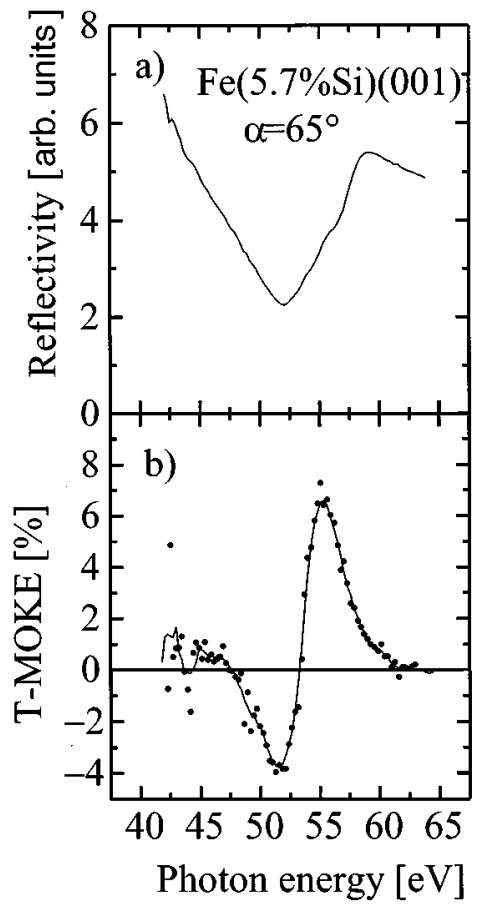

FIG. 1. (a) Reflectivity of bulk $\mathrm{Fe}(5.7 \% \mathrm{Si})(001)$ at $65^{\circ}$ incidence angle. (b) Asymmetry in the reflectivity upon magnetization reversal (transverse MOKE).

by the penetration depth of the radiation of $20 \mathrm{~nm}$ which is obtained from the optical constants. ${ }^{8}$

Figure 2(a) shows the reflectivity in the vicinity of the $\mathrm{Fe}$ $3 p$ core level for an 8-monolayer-(ML-) thick Fe/ $\mathrm{Ag}(001)$ sample at four different angles of incidence. The strong dip in the reflectivity at the core level threshold is also seen in this data. However, the total change in the reflectivity in the covered energy range is roughly only $1 / 2$ of that observed for the bulk sample. Obviously, the Fe-specific dependence of the reflectivity on the photon energy is weakened due to the small film thickness and the presence of the $\mathrm{Ag}$ substrate. Figure 2(b) shows the T-MOKE asymmetry. It is sharply peaked at the energy where the reflectivity has its minimum value. The T-MOKE signal is largest at $68^{\circ}$ incidence angle and amounts to $3 \%$ p.-p. Figure 2(c) shows the photon energy dependence of the total photoyield (sample current) together with its asymmetry. This data have been taken simultaneously with the reflectivity measurements. Figure 3 (solid line) shows the peak-to-peak asymmetries as a function of incidence angle. The asymmetry decreases strongly towards more grazing incidence. According to Fresnel's equations, generalized to include magneto-optical effects, ${ }^{1}$ the asymmetry should peak near $45^{\circ}$.

The transverse MOKE in the soft-x-ray regime can also be used to monitor the onset of in-plane long-range ferromagnetic order as a function of film thickness. Data obtained on a 5-ML-thick Fe film on $\mathrm{Ag}(001)$ (not shown) clearly exhibit a T-MOKE signal but the p.-p. value is only $0.4 \%$. The maximum value occurs at a slightly different photon energy as compared to the 8-ML-thick sample. In a film only 3 ML thick the T-MOKE asymmetry is absent. This is in accordance with the earlier observation of the onset of inplane anisotropy at 5 ML film thickness. ${ }^{9}$

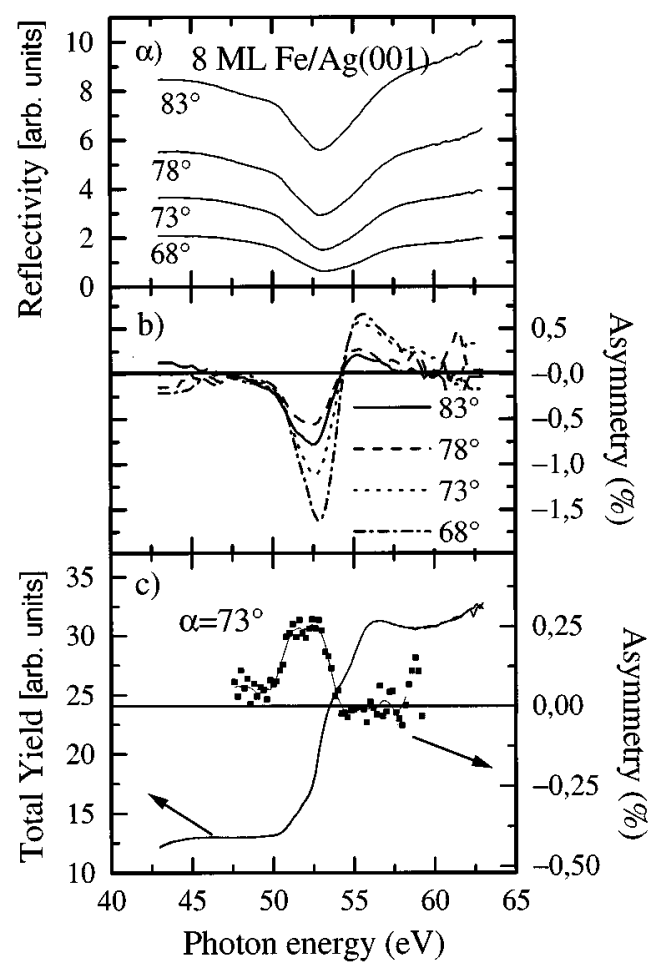

FIG. 2. (a) Reflectivity of an 8-ML-thick Fe film on $\mathrm{Ag}(001)$. (b) Asymmetry of the reflectivity (transverse MOKE) as a function incidence angle, obtained with $p$-polarized radiation. (c) Total photoyield, measured simultaneously with the reflectivity, and magnetic linear dichroism in the total photoyield.

The asymmetries in the reflectivity $(R)$ and in the total photoyield $(A)$ are closely related by the equation ${ }^{5}$

$$
\Delta A / A=-\Delta R / R \times R /(1-R),
$$

which stems from the relation $A+R=1$ if the intensity transmitted through the surface is eventually absorbed as in the case of an infinitely thick film. This form of the relation between $\Delta A$ and $\Delta R$ has the advantage of connecting experi-



FIG. 3. Peak-to-peak amplitudes of the T-MOKE (dashed line, left scale), the magnetic linear dichroism in the total photoyield (solid line, right scale), and $\Delta A / A$ as calculated from Eq. (1) (dashdotted line, right scale). 
mentally more easily accessible quantitites $(\Delta A / A)$ and $\Delta R / R$ with the absolute value of the reflectivity, which can also be measured or calculated from tabulated optical constants. ${ }^{8}$ According to Eq. (1), the dichroism should show an opposite behavior as a function of photon energy, as observed experimentally; see Figs. 2(b) and 2(c).

Figure 3 shows the angular dependences of the transverse MOKE and of the dichroism in the total photoyield. Also these dependences are roughly opposite to each other. However, due to the term $R /(1-R)$ in Eq. (1) which diverges as $R$ approaches 1 at grazing incidence, the dichroism in the total photoyield strongly increases towards grazing incidence whereas the T-MOKE should decrease towards zero at grazing incidence from its peak near $45^{\circ}$. The dip at $74^{\circ}$ might be due to interference effects related to the presence of a film/substrate system. The dichroism $\triangle A / A$ in the total photoyield as calculated from Eq. (1) is also shown in Fig. 3. Here the measured T-MOKE asymmetry $(\Delta R / R)_{\text {expt }}$ was multiplied by $R_{\mathrm{Fe}, B} /\left(1-R_{\mathrm{Fe}, B}\right)$ where $R_{\mathrm{Fe}, B}$ was calculated from tabulated optical constants for bulk Fe. ${ }^{8}$ The asymmetry obtained that way is smaller by a factor of 2 as compared to the experimental data for the five angles of incidence. This can be explained as follows: The reflectivity of the combined $\mathrm{Fe}$-vacuum and $\mathrm{Fe}$-substrate interface systems is given by the standard Fresnel coefficients. The reflectivity is then modified by multiple reflections at the interfaces. If the film is very thin, as in our case, the optical path length across the film is small compared to the light wavelength and accordingly interference effects are small. Therefore the reflection from the Fe-Ag interface adds a slowly varying background to the reflectivity of the $\mathrm{Fe}$ surface. A rough estimate based on the attenuation length of $10^{2} \AA$ in the minimum of the reflectivity as obtained from optical constants ${ }^{8}$ for the grazing incidence angle yields a contribution to the reflectivity from the Ag substrate of about 50\%, consistent with the observation [cf. Fig. 2(a)]. This is probably a general effect for $\mathrm{x}$-ray MOKE studies of thin films on metallic substrates (the quantitative value for the reduction of the reflectivity dip depending on the film thickness and the optical constants). Since the first term in Eq. (1) contains the measured combined reflectivity of the $\mathrm{Fe} / \mathrm{Ag}$ system rather than the reflectivity of bulk $\mathrm{Fe}$ as it enters the second term, the calculated asymmetry comes out reduced, approximately by a factor of 2 in the present case. Therefore, we can conclude that in a thin film system, the magnetic dichroism in the total photoyield is reduced from its value expected from the transverse MOKE of the bulk system. At more grazing incidence or for a film thickness comparable to the penetration depth of the light the influence of the substrate will be smaller.

In conclusion, we present transverse MOKE data obtained from a bulk $\mathrm{Fe}(5.7 \% \mathrm{Si})(001)$ sample in the vicinity of the $\mathrm{Fe} 3 p$ core level threshold. The asymmetry is similarly large as in previous data obtained on $\mathrm{Co}(001)$ films. We also present T-MOKE data on ultrathin Fe films on $\operatorname{Ag}(001)$ as functions of incidence angle and film thickness. With the simultaneously measured magnetic dichroism of the total photoyield we investigate its relationship to the transverse magneto-optical Kerr effect.

We wish to thank R. Treusch from HASYLAB who provided the Fe-Si single crystal together with his experience of the optimized cleaning and magnetization procedures. This work was supported by the Bundesminister für Bildung, Wissenschaft und Forschung, Contract No. 05\644\GUA9 and No. 05\644\PFA, by the Deutsche Forschungsgemeinschaft (SFB 166), and by special grants from the Minister für Wissenschaft und Forschung of Nordrhein Westfalen. One of us (I.R.) wishes to thank the DAAD for their support.
${ }^{1}$ M.J. Freiser, IEEE Trans. Magn. MAG-4, 152 (1968).

${ }^{2}$ K. Namikawa, M. Ando, T. Nakajima, and H. Kawata, J. Phys. Soc. Jpn. 54, 4099 (1985).

${ }^{3}$ C.C. Kao, J.B. Hastings, E.D. Johonson, D.P. Siddons, G.C. Smith, and G.A. Prinz, Phys. Rev. Lett. 65, 373 (1990).

${ }^{4}$ J.B. Kortright, M. Rice, and B. Carr, Phys. Rev. B 51, 10240 (1995).

${ }^{5}$ F.U. Hillebrecht et al., Phys. Rev. Lett. 75, 2224 (1995); T. Kinoshita et al., J. Electron Spectrosc. Relat. Phenom. 78, 237 (1996).

${ }^{6}$ J. Voss, M. Fornefett, C. Kunz, A. Moewes, M. Pretorius, A. Ranck, M. Schroeder, and V. Wedemeier, J. Electron. Spectrosc.
Relat. Phenom. 80329 (1996), and references therein.

${ }^{7}$ J. Voss, M. Fornefett, C. Kunz, A. Moewes, M. Pretorius, A. Ranck, M. Schroeder, and V. Wedemeier, J. Electron Spectrosc. Relat. Phenom. 80, 329 (1996); M. Pretorius, K. B. von Rautenfeld, M. Fornefett, J. Friedrich, M. Schroeder, H. Sievers, A. Ranck, J. Voss, M. Wachsmuth, and V. Wedemeier (unpublished).

${ }^{8}$ Handbook of Optical Constants of Solids, edited by E.D. Palik (Academic, Boston, 1991), Vol. II, p. 385ff; B.L. Henke et al., At. Data Nucl. Data Tables 27, 1 (1982).

${ }^{9}$ B.T. Jonker , K.-H. Walker, E. Kisker, G.A. Prinz, and C. Carbone, Phys. Rev. Lett. 57, 142 (1986). 